\title{
BMJ Open Safety and effectiveness of low-dose aspirin for the prevention of gastrointestinal cancer in adults without atherosclerotic cardiovascular disease: a population-based cohort study
}

\author{
Jessica J P Shami (D) , ' Jiaxi Zhao, ${ }^{1,2}$ Swathi Pathadka, ${ }^{1}$ Eric Yuk Fai Wan (D) , ${ }^{1,3,4}$ \\ Joseph Edgar Blais (D) , ${ }^{1}$ Pareen Vora, ${ }^{5}$ Montse Soriano-Gabarró, ${ }^{6}$ \\ Ka Shing Cheung, ${ }^{7}$ W K Leung, ${ }^{7}$ lan C K Wong, ${ }^{1,3,8}$ Esther W Chan ${ }^{1,3,9,10}$
}

To cite: Shami JJP, Zhao J, Pathadka S, et al. Safety and effectiveness of low-dose aspirin for the prevention of gastrointestinal cancer in adults without atherosclerotic cardiovascular disease: a population-based cohort study. BMJ Open 2022;12:e050510. doi:10.1136/ bmjopen-2021-050510

- Prepublication history and additional supplemental material for this paper are available online. To view these files, please visit the journal online (http://dx.doi.org/10.1136/ bmjopen-2021-050510)

Received 22 February 2021 Accepted 05 January 2022
Check for updates

(c) Author(s) (or their employer(s)) 2022. Re-use permitted under CC BY-NC. No commercial re-use. See rights and permissions. Published by BMJ.

For numbered affiliations see end of article.

Correspondence to

Dr Esther W Chan

ewchan@hku.hk

\section{ABSTRACT}

Objective To assess the association between lowdose aspirin and the incidence of colorectal cancer (CRC), gastric cancer (GC), oesophageal cancer (EC) and gastrointestinal bleeding (GIB) in adults without established atherosclerotic cardiovascular disease.

Design Cohort study with propensity score matching of new-users of aspirin to non-users.

Setting Clinical Data Analysis and Reporting System database, Hong Kong.

Participants Adults $\geq 40$ years with a prescription start date of either low-dose aspirin (75-300 mg/daily) or paracetamol (nonaspirin users) between 1 January 2004 to 31 December 2008 without a history of atherosclerotic cardiovascular disease. Main outcome measures The primary outcome was the first diagnosis of gastrointestinal cancer (either CRC, GC or EC) and the secondary outcome was GIB. Individuals were followed from index date of prescription until the earliest occurrence of an outcome of interest, an incident diagnosis of any type of cancer besides the outcome, death or until 31 December 2017. A competing risk survival analysis was used to estimate HRs and $95 \% \mathrm{Cls}$ with death as the competing risk.

Results After matching, 49679 aspirin and non-aspirin users were included. The median (IQR) follow-up was 10.0 (6.4) years. HRs for low-dose aspirin compared with non-aspirin users were 0.83 for $\mathrm{CRC}(95 \% \mathrm{Cl}, 0.76$ to 0.91$), 0.77$ for $\mathrm{GC}$ $(95 \% \mathrm{Cl}, 0.65$ to 0.92$)$ and 0.88 for $\mathrm{EC}(95 \% \mathrm{Cl}, 0.67$ to 1.16$)$. Patients prescribed low-dose aspirin had an increased risk of $\mathrm{GIB}(\mathrm{HR} 1.15,95 \% \mathrm{Cl}, 1.11$ to 1.20$)$, except for patients prescribed proton pump inhibitors or histamine $\mathrm{H} 2$-receptor antagonists (HR 1.03, 95\% Cl, 0.96 to 1.10).

Conclusion In this cohort study of Chinese adults, patients prescribed low-dose aspirin had reduced risks of CRC and GC and an increased risk of GIB. Among the subgroup of patients prescribed gastroprotective agents at baseline, however, the association with GIB was attenuated.

\section{INTRODUCTION}

Colorectal cancer (CRC) is the second most common cause of cancer death with approximately 1.8 million new cases and 826000

\section{Strengths and limitations of this study}

- This is the first study to evaluate the association of low-dose aspirin with gastrointestinal cancer (ie, colorectal cancer, gastric cancer and oesophageal cancer) and gastrointestinal bleeding among Chinese adults without atherosclerotic cardiovascular disease.

- This population-based cohort study has a large sample size, long duration of follow-up and used electronic health records from an integrated healthcare system that captures aspirin prescriptions and cancer outcomes.

- Complete information, however, was not available for alcohol consumption, smoking status and body mass index, which could be associated with the outcomes of interest.

deaths worldwide in 2018. ${ }^{1}$ The incidence of CRC is estimated to rise to 2.2 million people by 2030, with 1.1 million CRC associated deaths. ${ }^{2}$ Apart from CRC, gastric cancer (GC) and oesophageal cancer (EC) also pose a public health threat worldwide, with approximately 1 million and 600000 new cases in 2018, respectively. ${ }^{3}$

Given the significant burden of gastrointestinal (GI) cancers, pharmacological intervention may play an important role in reducing their risk. The use of low-dose aspirin to prevent GI cancers is controversial with different studies showing inconsistent results. ${ }^{4-7}$ Evidence from randomised clinical trials (RCTs) is the 'gold standard' for assessing the efficacy of treatments. Although no trial has specifically assessed low-dose aspirin for the prevention of GI cancers, a patient-level meta-analysis of aspirin trials suggests an association with a reduced risk 
of CRC after long-term follow-up. ${ }^{8}$ In addition to trial evidence, pooling of observational studies also demonstrate an association with a reduced risk of GI cancers. ${ }^{9}$ Given the accumulating evidence of benefit for low-dose aspirin, the US Preventative Services Task Force currently recommends initiation of low-dose aspirin for the primary prevention of atherosclerotic cardiovascular disease (ASCVD) and CRC, only for patients aged between 50 and 69 years with $\geq 10 \%$ 10-year risk of ASCVD who are not at an increased risk of bleeding. ${ }^{10}$

The risk-benefit ratio for low-dose aspirin, however, may differ by ethnicity. A recent study showed that the protective effects of aspirin on CRC varied among ethnicities with the strongest association of benefit observed among Caucasians. ${ }^{11}$ Furthermore, low-dose aspirin modestly increases the risk of gastrointestinal bleeding (GIB),${ }^{12}$ which might outweigh the GI cancer prevention benefits. The risk of GIB is especially a concern among the Chinese population as they are suspected to have a higher risk of bleeding. ${ }^{1314}$ Considering the possible variation in the effects of low-dose aspirin on GI cancer, as well as in the risk of GIB, further studies conducted in Asian populations are warranted.

This study aimed to investigate the association of lowdose aspirin with the risk of CRC, GC, EC and GIB among adults $\geq 40$ years without pre-existing ASCVD in Hong Kong.

\section{METHODS}

\section{Data source}

We used the Clinical Data Analysis and Reporting System (CDARS), which contains electronic health records for patients receiving care from the Hospital Authority (HA), a statutory body that manages all public hospitals and their clinics in Hong Kong. All Hong Kong residents have access to public healthcare services and around 80\% of hospitalisations in Hong Kong are in HA hospitals. CDARS stores clinical records from 1993 and has been used to conduct pharmacoepidemiological studies, with high accuracy in coding the study outcomes in previous validation studies (positive predictive value: GI bleed, $100 \%$; GI cancer, $100 \%) .^{15-17}$

\section{Study design and patient selection}

This was a population-wide retrospective cohort study. Patients $\geq 40$ years who were either prescribed low-dose aspirin $(75-300 \mathrm{mg} /$ daily) or paracetamol by a doctor within the HA, and with a prescription start date between 1 January 2004 and 31 December 2008 were identified in CDARS. The date of the first low-dose aspirin or paracetamol prescription was considered the index date. Since CDARS captures both prescribing and dispensing with the HA system, the prescription start date matched the dispensing date for $99 \%$ of the prescription records in our data set. To include new users of low-dose aspirin, patients with a prescription of aspirin 1 year prior to the index date were excluded. Patients diagnosed with any type of cancer, those who underwent a colectomy or gastrectomy or diagnosed with ASCVD defined as ischaemic heart disease, cerebrovascular disease or peripheral artery disease before the index date were excluded. Nitrates and digoxin were used as proxies to indicate a history of ASCVD, hence, any patient with a nitrate or digoxin prescription in the year prior to the index date were also excluded (online supplemental tables 1 and 2).

Patients who received paracetamol (non-aspirin users) were identified as the reference group for risk comparison. Paracetamol was used to identify patients who have had contact with the healthcare system during the same calendar time period as the low-dose aspirin patients. Importantly, paracetamol is not indicated for any associated comorbidities and has no known association with any type of cancer. An intention-to-treat approach was adopted, where patients allocated to the low-dose aspirin group on the index date will remain in the low-dose aspirin group, and similarly for the non-aspirin group.

\section{Outcomes}

The primary outcomes of this study were the development of either CRC, GC or EC. The follow-up period started from the date of first prescription of either lowdose aspirin or paracetamol (ie, index date) and was censored at the incident diagnosis of any cancer, death or end of study period (31 December 2017). Patients diagnosed with CRC, GC and EC were identified using International classification of diseases ninth revision codes (online supplemental table 1). The secondary outcome was GIB that led to a hospital visit (diagnosis code for an inpatient, outpatient or accident and emergency room visit). The follow-up period started from the index date and was censored at diagnosis of the outcome, death or end of study period.

\section{Study variables}

Potential confounders included patient demographics (age and sex), comorbidities (diabetes mellitus, hyperlipidaemia, hypertension, obesity, alcohol-related disorders, congestive heart failure, arrhythmia and conduction disorders, arterial disease, valve disorders, cardiomyopathy, chronic kidney disease, hepatic failure, chronic obstructive pulmonary disease (COPD), thyroid disorders, schizophrenia, depression, bipolar disorder, peptic ulcer, GI reflux, irritable bowel syndrome, inflammatory bowel syndrome and bleeds that led to hospitalisation within 1 year prior to index date) and concomitant medication use 1 year prior to index date (non-steroidal antiinflammatory drugs, antiplatelets, anticoagulants, oral hypoglycaemic agents, insulin, diuretics, antihypertensive agents, antiarrhythmic, calcium channel blockers, betablockers, angiotensin II receptor blocker/angiotensinconverting enzyme inhibitor, peripheral vasodilators, lipid-lowering drugs, oral bisphosphonates, oral corticosteroids, proton pump inhibitors (PPI)/histamine-2 receptor blockers (H2-blockers), antidepressants and antipsychotics). 
Although evidence indicates a potential chemoprotective role of estrogens on the risk of certain cancers a prescription of estrogens (either as oral contraceptive or menopausal hormone) was not included as a study variable due to the small number of patients with oestrogen therapy $(233(0.47 \%)$ and $244(0.49 \%)$ in low-dose aspirin and paracetamol users, respectively).

\section{Statistical analysis}

Baseline characteristics of low-dose aspirin users and nonaspirin users were presented as frequencies (percentages) for categorical variables and as mean $( \pm \mathrm{SD})$ for continuous variables. To reduce confounding arising from baseline differences between low-dose aspirin and non-aspirin users, propensity score (PS) matching was performed. Aforementioned confounders were included in estimating the PS value. Patients using low-dose aspirin and paracetamol were matched at a 1:1 ratio using a nearest neighbour algorithm with a calliper of 0.01 . Standardised mean difference $(\mathrm{SMD})<0.1$ between treatment groups was considered acceptable.

The ratio of incidence per 1000 person-years of CRC, GC and EC among low-dose aspirin users and nonaspirin users was reported. The association of CRC, GC and EC with the use of low-dose aspirin was estimated using competing risk Cox regression with death as the competing risk, and HR with 95\% CI was reported. The association of GIB with the use of low-dose aspirin was estimated using a Cox regression and HR with 95\% CI was reported. The number needed to treat (NNT) and number needed to harm (NNH) was estimated using the equation; 1 /absolute risk reduction and 1 /absolute risk increase, respectively.

Subgroup analysis was performed to investigate the risk of GI cancer and GIB in low-dose aspirin and nonaspirin users with different age groups (40-49 years old, $50-59$ years old, $60-69$ years old, $70-79$ years old and $\geq 80$ years old). Since the use of gastroprotective agents (PPI/ H2-blockers) could reduce the risk of GIB in patients on antithrombotic agents, ${ }^{18}$ we assessed the association of GI cancer and GIB with the use of low-dose aspirin in patients on gastroprotective agents. As people with diabetes are at higher risk of developing cancer, ${ }^{19}$ we also evaluated the association of low-dose aspirin with GI cancer and GIB among this population. Lastly, the association between low-dose aspirin and GIB has been shown to be different depending on the location of the GIB. Therefore, we stratified the GIB outcome to upper GIB (UGIB) and lower GIB (LGIB).

Sensitivity analyses were conducted by excluding patients with cancer diagnosis during the first year of follow-up since the cancer could have developed before the start of follow-up. Patients with an ASCVD diagnosis during the first year of follow-up were removed to ensure all patients included have no pre-existing ASCVD. Nonaspirin users with a low-dose aspirin prescription during follow-up were censored at the first aspirin prescription. Lastly, the effectiveness of low-dose aspirin for GI cancer

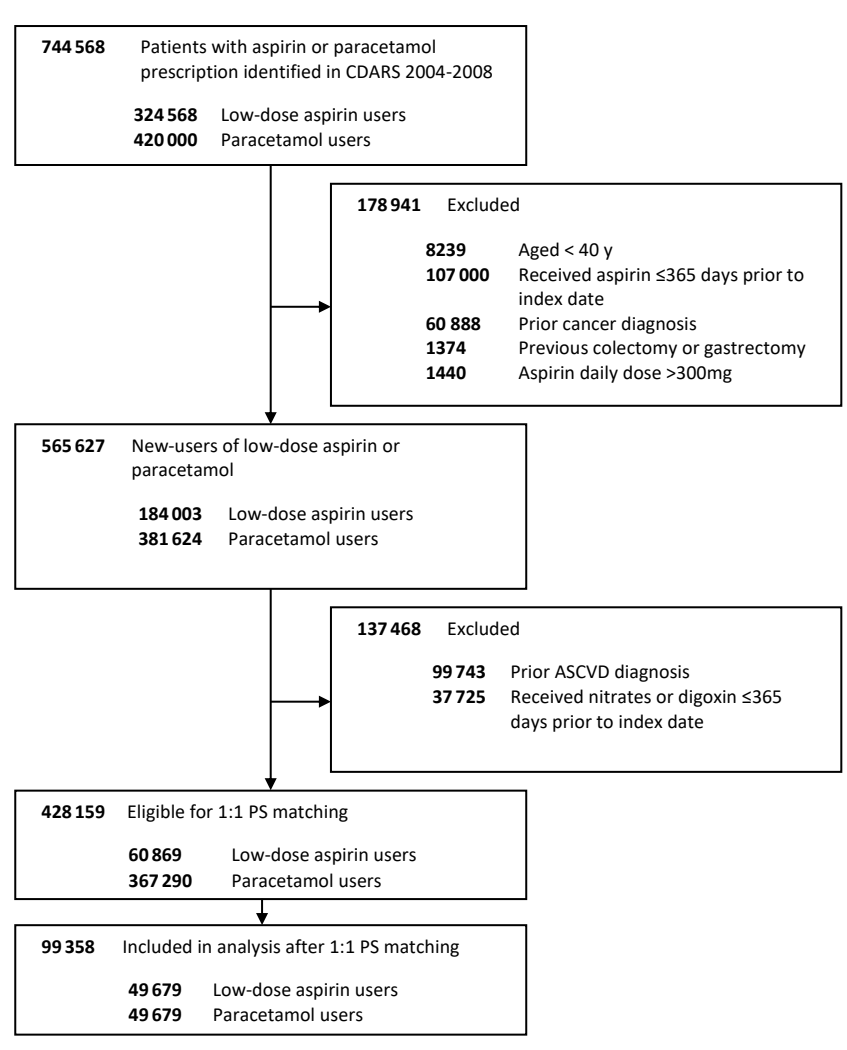

Figure 1 Flow chart of users in the cohort study assessing the risk of gastrointestinal cancer and gastrointestinal bleeding. ASCVD, atherosclerotic cardiovascular disease; CDARS, Clinical Data Analysis and Reporting System (of the Hong Kong Hospital Authority); d, days; PS, propensity score; $y$, years.

prevention was evaluated in patients taking low-dose aspirin for secondary ASCVD prevention; patients taking low-dose aspirin for primary and secondary ASCVD were included.

$\mathrm{R}$ 3.6.2 ( $\mathrm{R}$ Foundation for Statistical Computing) was used for all statistical analyses. The analyses were conducted by JJPS and cross-checked independently by JZ for quality assurance.

\section{Patient and public involvement}

There was no patient and public involvement.

\section{RESULTS}

\section{Baseline characteristics}

We identified 324568 aspirin and 420000 non-aspirin users between 1 January 2004 and 31 December 2008. Following exclusion criteria, 428159 patients were eligible for the PS matching (figure 1). A total of 99358 individuals (49 679 low-dose aspirin users and 49679 matched non-aspirin users) were successfully matched (online supplemental figure 1). After matching, all baseline 
characteristics had SMD $<0.1$ and were well balanced. The mean (SD) age for the cohort was 68.6 (12.6) years, and $48022(48.4 \%)$ were women (table 1$)$. The median (IQR) follow-up for the cohort was 10.0 (6.4) years for the GI cancer outcome (9.8 (6.3) years low-dose aspirin users and 10.4 (6.3) years non-aspirin users), and 10.2 (5.9) years for the GIB outcome (9.9 (6.1) years low-dose aspirin users and 10.6 (5.7) years non-aspirin users). The most common dose of aspirin was $80 \mathrm{mg}$ daily $(72.2 \%)$.

\section{Risk of Gl cancer}

In the propensity score-matched sample, 1954 of 99358 participants developed CRC (876 low-dose aspirin users $(1.7 \%)$ and 1078 non-aspirin users (2.2\%)), 515 GC (222 $(0.4 \%)$ and $293(0.6 \%))$ and 206 EC $(96(0.2 \%)$ and $110(0.2 \%)$, respectively; table 2$)$. The results for the unmatched cohort are presented in online supplemental table 3. The number of patients who died due to CRC, GC and EC were $247(28.2 \%), 99(44.6 \%)$ and $51(53.1 \%)$ in low-dose aspirin users, respectively, and 360 (33.4\%), $151(51.5 \%)$ and $55(50.0 \%)$ in non-aspirin users, respectively. NNT is 250 and 500 for CRC and GC, respectively, and the NNH is 125 for GIB.

The results from the competing risk survival analysis showed that low-dose aspirin use was significantly associated with a lower risk of CRC and GC compared with nonaspirin users (CRC: HR, 0.83 (95\% CI, 0.76 to 0.91); GC: HR, 0.77 (95\% CI, 0.65 to 0.92$)$ ), but not with EC (HR, 0.88 (95\% CI, 0.67 to 1.16 ); table 2 ). The association with lower risk was statistically significant for women (CRC: HR, 0.79 (95\% CI, 0.68 to 0.90); GC: HR, 0.73 (95\% CI, 0.54 to 0.98$)$ ) and men (CRC: HR, 0.86 (95\% CI, 0.76 to 96$)$; GC: HR, 0.79 (95\% CI, 0.64 to 0.98$)$ ). The use of low-dose aspirin was significantly associated with a lower risk of CRC in patients aged between 70 and 79 years old (HR, 0.82 (95\% CI, 0.71 to 0.94$)$ ) and among patients with diabetes (HR, 0.73 (95\% CI, 0.57 to 0.94$)$ ), with a lower risk of GC among patients 80 years and older (HR, 0.60 (95\% CI, 0.43 to 0.84 ); table 2 ).

There was no significant association between low-dose aspirin and EC in any of the subgroup analysis (table 2). The test for the interaction effect of low-dose aspirin and gastroprotective agents was not significant when assessing the association between low-dose aspirin and GI cancer, with and without gastroprotective agents ( $\mathrm{p}$ value for interaction, $>0.5$ ).

\section{Risk of Gl bleeding}

In the propensity score-matched sample, 10629 of 99358 participants had a GIB event (5498 low-dose aspirin users $(11.1 \%)$ and 5131 non-aspirin users $(10.3 \%)$; table 3$)$. Among patients with a GIB diagnosis the number of patients who died due to a GIB was $88(1.6 \%)$ in lowdose aspirin users and $83(1.6 \%)$ in non-aspirin users. Compared with non-aspirin users, low-dose aspirin was significantly associated with an increased risk of GIB (HR, 1.15 (95\% CI, 1.11 to 1.20$)$ ). The association with higher risk was statistically significant for women (HR, 1.16 (95\%
CI, 1.10 to 1.23$)$ ) and men (HR, 1.15 (95\% CI, 1.09 to $1.21)$ ), in addition to patients aged 60-69 (HR, 1.13 (95\% CI, 1.03 to 1.23$)$ ), 70-79 (HR, 1.44 (95\% CI, 1.35 to 1.53$)$ ) and 80 years and older (HR, 1.18 (95\% CI, 1.11 to 1.27 ).

Low-dose aspirin was not significantly associated with an increased risk of GIB in patients aged 40-49 (HR, 0.94 (95\% CI, 0.77 to 1.15$)$ ) and 50-59 (HR, 1.05 (95\% CI, 0.93 to 1.19$)$ ) as well as in patients with diabetes (HR, 1.07 (95\% CI, 0.97 to 1.18 )) and those taking gastroprotective agents (HR, 1.03 (95\% CI, 0.96 to 1.10 ); table 3). The test for subgroup difference indicated significant difference between the association with and without gastroprotective agents ( $p$ value for interaction $<0.001$ ) (online supplemental table 4).

Low-dose aspirin was significantly associated with an increased risk of UGIB (HR, 1.14 (95\% CI, 1.09 to 1.18)) and LGIB (HR, 1.31 (95\% CI, 1.16 to 1.48)). The association with higher risk remained for LGIB among patients taking gastroprotective agents (HR, 1.70 (95\% CI, 1.35 to 2.14)), however, low-dose aspirin was not associated with an increased risk of UGIB in those taking gastroprotective agents (HR, 0.98 (95\% CI, 0.91 to 1.05)).

\section{Sensitivity analysis}

After removing patients with a cancer diagnosis during the first year of follow-up, the association remained similar for CRC (HR, 0.88 (95\% CI, 0.80 to 0.96)), GC (HR, $0.76(95 \%$ CI, 0.63 to 0.93$)$ ) and EC (HR, 1.13 (95\% CI, 0.83 to 1.55 ); figure 2 ). The association with lower risk also remained after removing patients with a diagnosis of ASCVD during the first year of follow-up for CRC (HR, 0.90 (95\% CI, 0.82 to 0.99$)$ ), GC (HR, 0.78 (95\% CI, 0.66 to 0.94$)$ ) and EC (HR, 0.70 (95\% CI, 0.53 to 0.94$)$ ). Lastly, the lower risk remained when censoring non-aspirin users at the first aspirin prescription during follow-up in CRC (HR, 0.88 (95\% CI, 0.80 to 0.96 )), and GC (HR, 0.80 (95\% CI, 0.67 to 0.96$)$ ) but not EC (HR, 0.93 (95\% CI, 0.71 to 1.23$)$ ). After combining all patients taking low-dose aspirin for either primary or secondary prevention of ASCVD, they had a lower risk of CRC (HR, 0.89 (95\% CI, 0.83 to 0.96$)$ ), GC (HR, 0.78 (95\% CI, 0.69 to 0.89$)$ ), as well as EC (HR, 0.73 (95\% CI, 0.60 to 0.90$)$ ) compared with non-aspirin users.

\section{DISCUSSION}

In Chinese adults without pre-existing ASCVD, our results suggest that the use of low-dose aspirin was associated with a lower risk of CRC and GC, but not EC, as compared with non-aspirin users during a median follow-up of 10 years. However, low-dose aspirin was associated with an increased risk of GIB. Nevertheless, a subgroup analysis showed that the use of low-dose aspirin was not associated with an increased risk of GIB among patients younger than 60 years old and those taking PPIs or H2-blockers.

Our findings are consistent with a meta-analysis of patient follow-up (maximum duration 20 years) from five RCTs which showed that aspirin was associated with a 
Table 1 Baseline characteristics of low-dose aspirin and paracetamol users

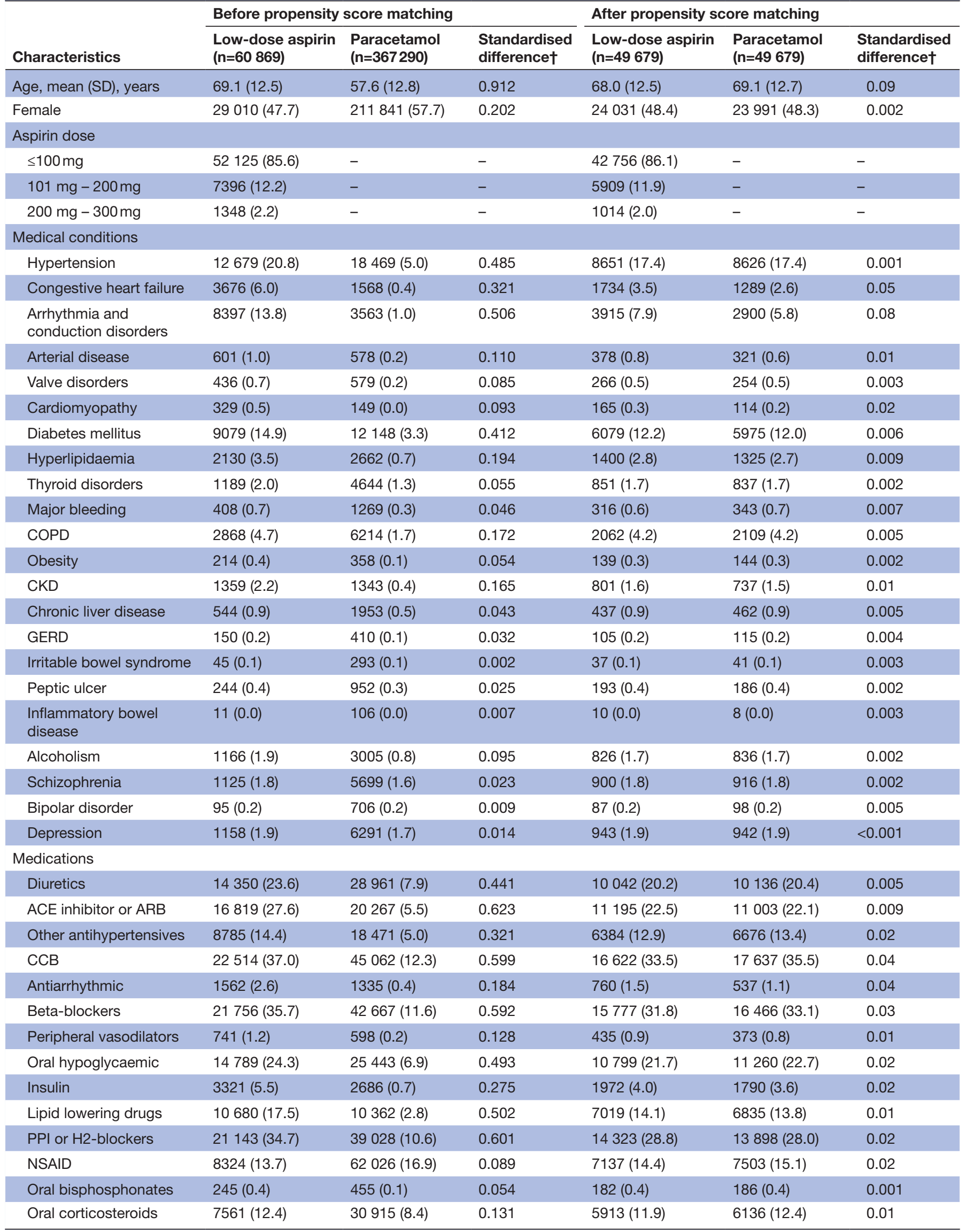


Table 1 Continued

\begin{tabular}{|c|c|c|c|c|c|c|}
\hline \multirow[b]{2}{*}{ Characteristics } & \multicolumn{3}{|c|}{ Before propensity score matching } & \multicolumn{3}{|c|}{ After propensity score matching } \\
\hline & $\begin{array}{l}\text { Low-dose aspirin } \\
(\mathrm{n}=60 \text { 869) }\end{array}$ & $\begin{array}{l}\text { Paracetamol } \\
(\mathrm{n}=367290)\end{array}$ & $\begin{array}{l}\text { Standardised } \\
\text { difference }\end{array}$ & $\begin{array}{l}\text { Low-dose aspirin } \\
(\mathrm{n}=49679)\end{array}$ & $\begin{array}{l}\text { Paracetamol } \\
(n=49679)\end{array}$ & $\begin{array}{l}\text { Standardised } \\
\text { difference } †\end{array}$ \\
\hline Antiplatelet & $1408(2.3)$ & $328(0.1)$ & 0.205 & $532(1.1)$ & $316(0.6)$ & 0.05 \\
\hline Antipsychotics & $2172(3.6)$ & $7718(2.1)$ & 0.088 & $1664(3.3)$ & $1708(3.4)$ & 0.005 \\
\hline
\end{tabular}

*Values are expressed as frequency (\%) unless otherwise specified.

†Standardised difference indicates difference in mean or proportion of covariates in the low-dose aspirin group versus the paracetamol group divided by the pooled SD.

ACE, angiotensin-converting enzyme; ARB, angiotensin II receptor blocker; CCB, calcium channel blocker; CKD, chronic kidney disease; COPD, chronic obstructive pulmonary disease; GERD, gastro-oesophageal reflux disease; H2-blockers, histamine-2 receptor blockers; NSAID, non-steroidal anti-inflammatory drug; PPI, proton pump inhibitors.

reduced risk of colorectal cancer (HR, $0.76 ; 95 \% \mathrm{CI}, 0.60$ to 0.96$).{ }^{8}$ In addition to RCTs, observational studies have also examined the association of low-dose aspirin with GI cancer. ${ }^{20-27}$ Although studies have consistently shown a beneficial effect of using low-dose aspirin, findings from both RCTs and observational studies have largely been limited to Caucasians. ${ }^{20} 22-2427$ An earlier study in Hong Kong evaluated the risk of GIB and benefit of CRC reduction from the use of low-dose aspirin and found that lowdose aspirin lowered the risk of CRC but at the cost of a higher risk of GIB. The authors acknowledged that the results could be inaccurate due to confounding by indication since no comorbidities were used to adjust for baseline differences between aspirin and non-aspirin users. ${ }^{28}$ Our present study adjusted for observed baseline differences between aspirin and non-aspirin users by using PS matching. Moreover, most studies include patients taking low-dose aspirin for both primary and secondary prevention of ASCVD. However, the clinical implications for the primary prevention cohort is greater as initiating low-dose aspirin is no longer standard practice for this population.

A study in the UK has evaluated the protective effect of low-dose aspirin on CRC in a cohort with no pre-existing CVD. ${ }^{4}$ However, the risk of GIB was not investigated. Nevertheless, the association of low-dose aspirin with a reduced risk of GI cancer was consistent with our findings. Furthermore, our findings are also consistent with our recent 13-year cohort study conducted in Hong Kong $(\mathrm{N}=74$ 161) which found that regular aspirin use was associated with a decrease in gastric cancer risk following Helicobacter pylori eradication. ${ }^{16}$ Daily use, prolonged use and use of higher doses of aspirin after $H$. pylori eradication was associated with significant reduction in the risk of GC. ${ }^{16}$

The role of low-dose aspirin for the prevention of GI cancer is equivocal and questions remain, particularly for patients without a history of ASCVD. Some RCTs have reported no reductions in GI cancer incidence and mortality with the use of low-dose aspirin. ${ }^{5} 7$ The Aspirin in Reducing Events in the Elderly (ASPREE) trial reported a higher mortality rate in patients taking low-dose aspirin compared with placebo. A secondary analysis showed cancer as the major contributor to the higher mortality rate (HR, 1.13; 95\% CI, 1.10 to 1.56 ), with a subgroup analysis for GI cancer which detected no differences between groups (CRC: RR, 0.97 (95\% CI, 0.77 to 1.24)). ${ }^{6}$ Patients in the ASPREE trial were $\geq 70$ years old, hence the benefits of low-dose aspirin for GI cancer prevention may be limited since most of the benefits of low-dose aspirin are apparent in studies of younger adults with longer duration of use. ${ }^{29}$ Notably, Asians comprised only $1 \%$ of the trial population in ASPREE. Therefore, findings from ASPREE may be more applicable to healthy Caucasian adults.

\section{Potential clinical implications}

The finding that low-dose aspirin use was associated with a lower risk of CRC and GC is of particular clinical importance, especially among patients with no pre-existing ASCVD, since the decision to initiate low-dose aspirin is less well defined. GI cancers are major contributors to mortality worldwide with no proven preventative treatment. Aspirin is affordable, easily accessible and has a recognised pharmacological profile which could be a means to improving the burden of disease. Additionally, the risk of GIB associated with low-dose aspirin is of particular interest in the Chinese population, which has a different bleeding profile compared with Caucasians. ${ }^{14}$ Lastly, our study showed that for every 1000 patients taking low-dose aspirin, 6GI cancer cases could be prevented, although it could cause 8 GIBs. However, the percentage of patients with GI cancer outcome who died was $30 \%-50 \%$ compared with $1.6 \%$ for GIB. In addition, the percentage of fatal GIB $(1.6 \%)$ is similar in both the low-dose aspirin and non-aspirin group. This indicates that the use of low-dose aspirin does not contribute to an increase in the risk of fatal GIB. Further, this is consistent with a meta-analysis published in 2016 which evaluated fatal GIB attributable to low-dose aspirin. ${ }^{30}$ This information along with the knowledge that patients under 60 years or those taking gastroprotective agents are not at higher risk of GIB could inform clinical decisions to 


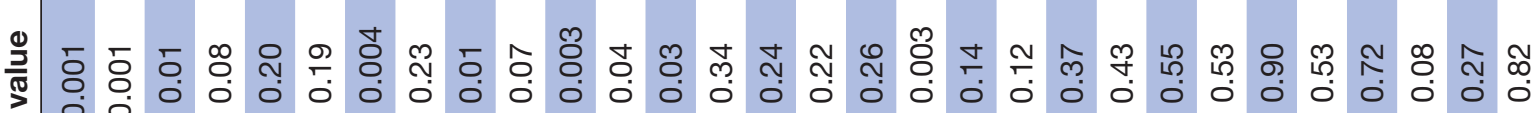
a $\mathrm{V}$ V

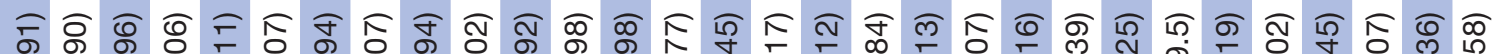

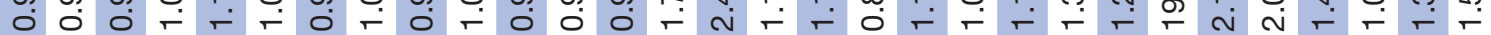

등ㅇㅇㅇㅇㅇㅇㅇㅇㅇㅇㅇㅇㅇㅇㅇㅇㅇㅇㅇㅇㅇㅇㅇㅇㅇㅇㅇㅇㅇㅇㅇㅇㅇㅇㅇㅇㅇㅇㅇ

○

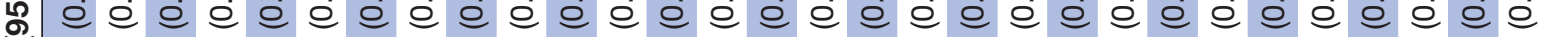

番

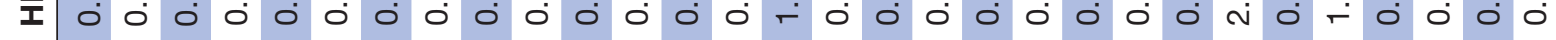

은

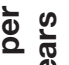

¿

응 은

일

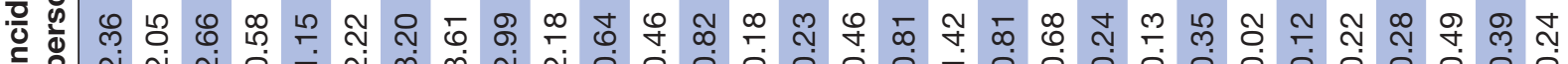

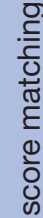

कृ

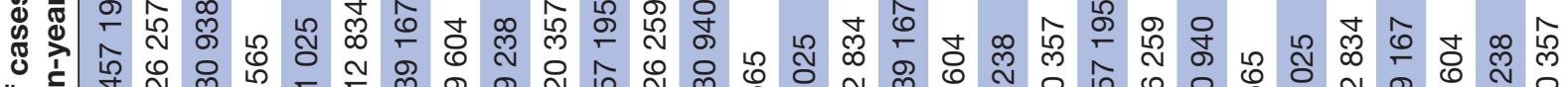

to

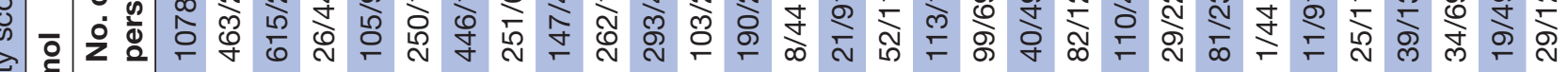

๑ 范

2 व 2

응

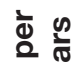

过

응

至

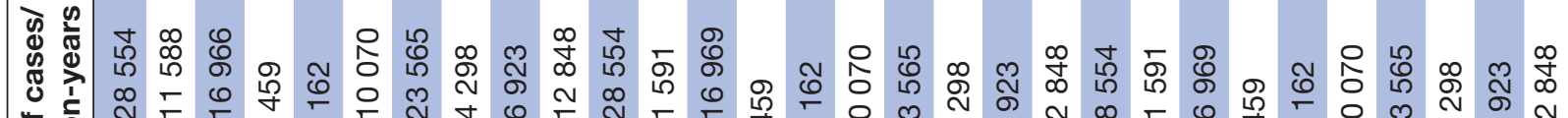

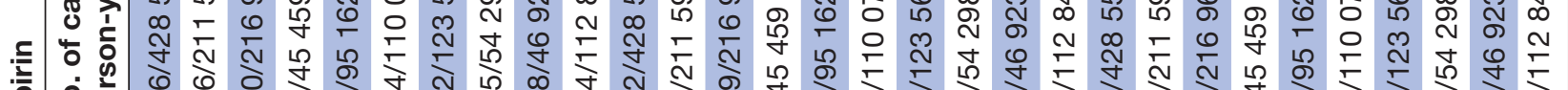

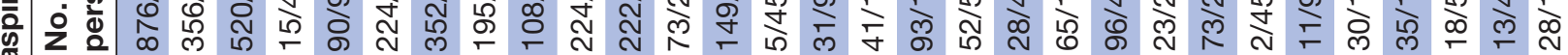

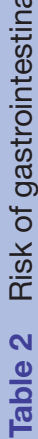

2 $\infty$ <

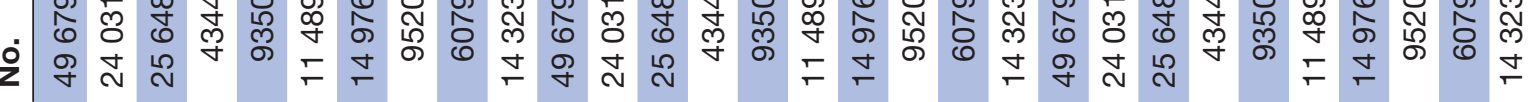

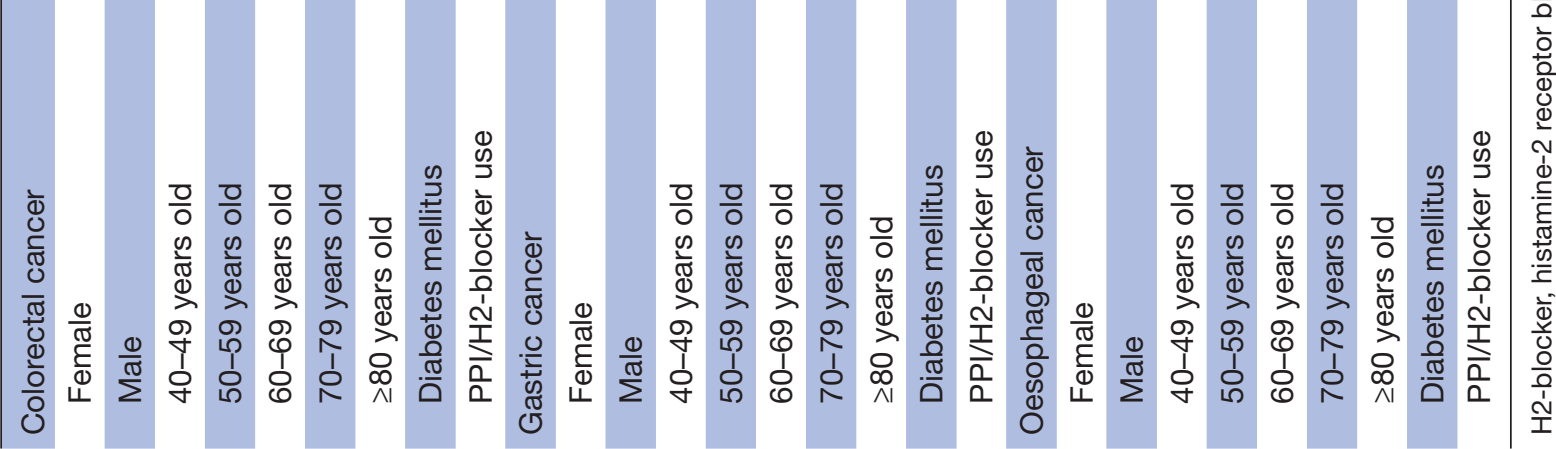




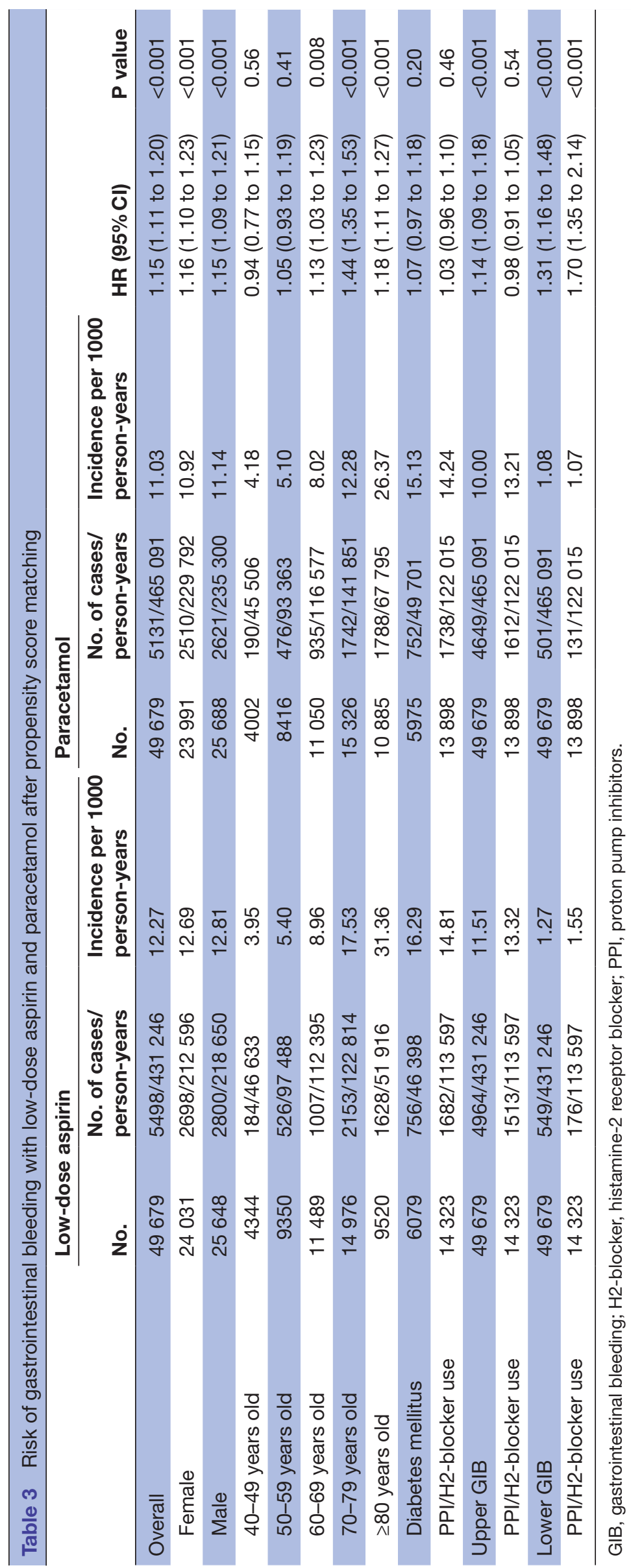




\section{Analysis}

\section{Colorectal cance}

Primary analysis

Cohort with ASCVD

Patients without cancers in 1st year

Patients without ASCVD in 1st year

Acetaminophen users censored at switching to aspirin

Gastric cancer

Primary analysis

Cohort with ASCVD

Patients without cancers in 1st year

Patients without ASCVD in 1st year

Acetaminophen users censored at switching to aspirin

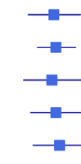

$0.77(0.65-0.92) \quad .003$

$0.78(0.69-0.89)<.001$

$0.76(0.63-0.93) \quad .007$

$0.78(0.66-0.94) \quad .008$

$0.80(0.67-0.96) \quad .01$

\section{Esophageal cancer}

Primary analysis

Cohort with ASCVD

Patients without cancers in 1 st year

Patients without ASCVD in 1st year

Acetaminophen users censored at switching to aspirin

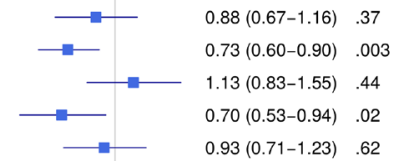

GI bleeding short-term outcomes

Primary analysis

1 Year follow up

3 Years follow up

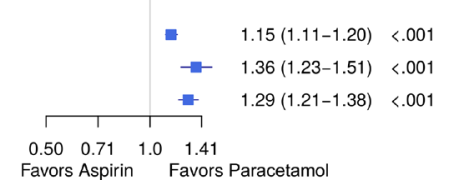

Figure 2 Forest plot of the results of the primary and sensitivity analyses. ASCVD, atherosclerotic cardiovascular disease; Gl, gastrointestinal.

initiate low-dose aspirin in Chinese adults without ASCVD who highly value preventing CRC and GC.

\section{Strengths and limitations}

To our knowledge, this is the first study to evaluate the association of low-dose aspirin with GI cancer and GIB among patients without ASCVD. The findings are likely generalisable to other urban Chinese populations with similar risks of GI cancer as the population of Hong Kong. We used PS matched cohort study to emulate a target randomised trial since the feasibility of an RCT is low due to the large sample size and long follow-up that is required to evaluate cancer outcomes. Furthermore, while low-dose aspirin is a non-prescription medication in Hong Kong, its cost is heavily subsidised ( $\mathrm{HK} \$ 15$ US\$2 for 4-month supply) through the public healthcare system. Thus, misclassification of exposure to low-dose aspirin is likely minimal. ${ }^{14}$

This study has several limitations. Similar to some electronic health record databases, information such as body mass index, smoking status and alcohol consumption are not routinely recorded in CDARS. However, other confounders were used as proxy to account for these risk factors (COPD and alcohol-related disorders). A general limitation of cohort studies is the residual and the unmeasured confounding bias which cannot be excluded. Finally, subgroup analyses by age, diabetes mellitus and use of gastroprotective agents should be interpreted as hypothesis generating results since the low number of events on stratification resulted in limited statistical power.
Our findings support a potential role for low-dose aspirin therapy for the prevention of CRC and GC, but not EC, in Chinese adults $\geq 40$ years. Further research, such as a pragmatic RCT, is needed to confirm the observed association in a patient population that would be expected to derive the most benefit, and least harm, from taking low-dose aspirin.

\section{Author affiliations}

${ }^{1}$ Centre for Safe Medication Practice and Research, Department of Pharmacology and Pharmacy, The University of Hong Kong, Hong Kong SAR, China

'Digital and Data Innovation, AstraZeneca Global R\&D (China) Co., Ltd, Shanghai, China

${ }^{3}$ Laboratory of Data Discovery for Health (D24H), Hong Kong Science and Technology Park, Hong Kong SAR, China

${ }^{4}$ Department of Family Medicine and Primary Care, University of Hong Kong, Hong Kong

${ }^{5}$ Epidemiology, Bayer AG, Leverkusen, Nordrhein-Westfalen, Germany

${ }^{6}$ Epidemiology, Bayer Pharma AG, Berlin, Germany

${ }^{7}$ Department of Medicine, University of Hong Kong, Hong Kong

${ }^{8}$ Centre for Medicines Optimisation Research and Education, University College

London Hospitals NHS Foundation Trust, London, UK

${ }^{9}$ Department of Pharmacy, The University of Hong Kong-Shenzhen Hospital,

Shenzhen, China

${ }^{10}$ Shenzhen Institute of Research and Innovation, The University of Hong Kong, Shenzhen, China

\section{Twitter Joseph Edgar Blais @blaisjoseph}

Contributors EWC and JJPS had full access to all of the data in the study and take responsibility for the integrity of the data, the accuracy of the data analysis and contolled the decision to publish. Concept and design: JJPS, JZ, EWC and ICKW. Acquisition, analysis or interpretation of data: JJPS, JZ, SP, EYFW, EWC and ICKW. Drafting of the manuscript: JJPS. Critical revision of the manuscript for important intellectual content: JJPS, JZ, SP, EYFW, JEB, KSC, WKL, ICKW and EWC. Statistical analysis: JJPS, JZ and EYFW. Administrative, technical or material support: PV, MSG, ICKW and EWC. Supervision: EWC and ICKW.

Funding This work was partially supported by Bayer AG - General Award (Grant number: RS170309). The sponsors had no role in the study design, data collection and analysis.

Competing interests EWC has received honorarium from the Hospital Authority and research funding from The Hong Kong Research Grants Council, The Research Fund Secretariat of the Food and Health Bureau, Narcotics Division of the Security Bureau of HKSAR, Hong Kong; National Natural Science Fund of China, China; Wellcome Trust, UK; Bristol Myers Squibb, Pfizer and Takeda, for work unrelated to this study. ICKW has received research funding outside the submitted work from the Hong Kong Research Grants Council and the Hong Kong Health and Medical Research Fund, National Institute for Health Research in the UK, European Commission, Amgen, Bayer, Bristol Myers Squibb, GSK and Janssen. PV and MS-G are employees of Bayer AG.

Patient consent for publication Not applicable.

Ethics approval This study was approved by the Institutional Review Board of the University of Hong Kong/Hospital Authority Hong Kong West Cluster (Reference number: UW 18-033). Informed patient consent was not required as the data used in this study were anonymised.

Provenance and peer review Not commissioned; externally peer reviewed.

Data availability statement No data are available. No additional data are available.

Supplemental material This content has been supplied by the author(s). It has not been vetted by BMJ Publishing Group Limited (BMJ) and may not have been peer-reviewed. Any opinions or recommendations discussed are solely those of the author(s) and are not endorsed by BMJ. BMJ disclaims all liability and responsibility arising from any reliance placed on the content. Where the content includes any translated material, BMJ does not warrant the accuracy and reliability of the translations (including but not limited to local regulations, clinical guidelines, terminology, drug names and drug dosages), and is not responsible for any error and/or omissions arising from translation and adaptation or otherwise. 
Open access This is an open access article distributed in accordance with the Creative Commons Attribution Non Commercial (CC BY-NC 4.0) license, which permits others to distribute, remix, adapt, build upon this work non-commercially, and license their derivative works on different terms, provided the original work is properly cited, appropriate credit is given, any changes made indicated, and the use is non-commercial. See: http://creativecommons.org/licenses/by-nc/4.0/.

\section{ORCID iDs}

Jessica J P Shami http://orcid.org/0000-0002-1084-1829

Eric Yuk Fai Wan http://orcid.org/0000-0002-6275-1147

Joseph Edgar Blais http://orcid.org/0000-0001-7895-198X

\section{REFERENCES}

1 World Cancer Research Fund. Colorectal cancer statistics. Available: https://www.wcrf.org/dietandcancer/cancer-trends/colorectalcancer-statistics [Accessed 4 Oct 2020].

2 Arnold M, Sierra MS, Laversanne M, et al. Global patterns and trends in colorectal cancer incidence and mortality. Gut 2017;66:683-91.

3 International Agency for Research on Cancer WHO. Cancer today 2018. Available: https://gco.iarc.fr/today/ [Accessed 12 Dec 2020].

4 Cea Soriano L, Soriano-Gabarró M, García Rodríguez LA. Incidence of colorectal cancer in new users and non-users of low-dose aspirin without existing cardiovascular disease: a cohort study using the health improvement network. Int J Cardiol 2017;248:376-81.

5 Cook NR, Lee I-M, Gaziano JM, et al. Low-Dose aspirin in the primary prevention of cancer: the women's health study: a randomized controlled trial. JAMA 2005;294:47-55.

6 McNeil JJ, Nelson MR, Woods RL, et al. Effect of aspirin on all-cause mortality in the healthy elderly. N Engl J Med 2018;379:1519-28.

7 Stürmer T, Glynn RJ, Lee IM, et al. Aspirin use and colorectal cancer: post-trial follow-up data from the physicians' health study. Ann Intern Med 1998;128:713-20.

8 Rothwell PM, Wilson M, Elwin C-E, et al. Long-Term effect of aspirin on colorectal cancer incidence and mortality: 20-year follow-up of five randomised trials. Lancet 2010;376:1741-50.

9 Bosetti C, Santucci C, Gallus S, et al. Aspirin and the risk of colorectal and other digestive tract cancers: an updated metaanalysis through 2019. Ann Oncol 2020;31:558-68.

10 U.S Preventive Services Task Force. aspirin use to prevent cardiovascular disease and colorectal cancer: preventive medication, 2016. Available: https://www.uspreventiveservicestaskforce.org/ Page/Document/RecommendationStatementFinal/aspirin-to-preventcardiovascular-disease-and-cancer [Accessed 4 Oct 2020].

11 Park S-Y, Wilkens LR, Kolonel LN, et al. Exploring differences in the aspirin-colorectal cancer association by sex and race/ethnicity: the Multiethnic cohort study. Cancer Epidemiol Biomarkers Prev 2017;26:162-9.

12 García Rodríguez LA, Martín-Pérez M, Hennekens $\mathrm{CH}$, et al. Bleeding risk with long-term low-dose aspirin: a systematic review of observational studies. PLoS One 2016;11:e0160046-e46.

13 Guo Y-T, Zhang Y, Shi X-M, et al. Assessing bleeding risk in 4824 Asian patients with atrial fibrillation: the Beijing PLA Hospital atrial fibrillation project. Sci Rep 2016;6:31755
14 Shen AY-J, Yao JF, Brar SS, et al. Racial/Ethnic differences in the risk of intracranial hemorrhage among patients with atrial fibrillation. $J$ Am Coll Cardiol 2007;50:309-15.

15 Chan EW, Lau WCY, Leung WK, et al. Prevention of dabigatranrelated gastrointestinal bleeding with gastroprotective agents: a population-based study. Gastroenterology 2015;149:586-95.

16 Cheung KS, Chan EW, Wong AYS, et al. Aspirin and risk of gastric cancer after Helicobacter pylori eradication: a territory-wide study. $J$ Natl Cancer Inst 2018;110:743-9.

17 Cheung KS, Chan EW, Wong AYS, et al. Long-term proton pump inhibitors and risk of gastric cancer development after treatment for Helicobacter pylori: a population-based study. Gut 2018;67:28-35.

18 Lin KJ, Hernández-Díaz S, García Rodríguez LA. Acid suppressants reduce risk of gastrointestinal bleeding in patients on antithrombotic or anti-inflammatory therapy. Gastroenterology 2011;141:71-9.

19 Ohkuma T, Peters SAE, Woodward M. Sex differences in the association between diabetes and cancer: a systematic review and meta-analysis of 121 cohorts including 20 million individuals and one million events. Diabetologia 2018;61:2140-54.

20 Akre K, Ekström AM, Signorello LB, et al. Aspirin and risk for gastric cancer: a population-based case-control study in Sweden. Br J Cancer 2001;84:965-8.

21 Cao Y, Nishihara R, Wu K, et al. Population-Wide impact of long-term use of aspirin and the risk for cancer. JAMA Oncol 2016;2:762-9.

22 Cea Soriano L, Soriano-Gabarró M, García Rodríguez LA. The protective effect of low-dose aspirin against colorectal cancer is unlikely explained by selection bias: results from three different study designs in clinical practice. PLoS One 2016;11:e0159179 -e79.

23 Friis $\mathrm{S}$, Riis $\mathrm{AH}$, Erichsen R, et al. Low-Dose aspirin or nonsteroidal anti-inflammatory drug use and colorectal cancer risk: a populationbased, case-control study. Ann Intern Med 2015;163:347-55.

24 Hollestein LM, van Herk-Sukel MPP, Ruiter R, et al. Incident cancer risk after the start of aspirin use: results from a Dutch populationbased cohort study of low dose aspirin users. Int J Cancer 2014;135:157-65.

25 Huang W-K, Chiou M-J, Yu K-H, et al. The association between lowdose aspirin use and the incidence of colorectal cancer: a nationwide cohort study. Aliment Pharmacol Ther 2013;38:432-9.

$26 \mathrm{Kim}$ Y-I, Kim SY, Kim JH, et al. Long-Term low-dose aspirin use reduces gastric cancer incidence: a nationwide cohort study. Cancer Res Treat 2016;48:798-805.

27 Sivarasan N, Smith G. Role of aspirin in chemoprevention of esophageal adenocarcinoma: a meta-analysis. J Dig Dis 2013;14:222-30.

28 Ajrouche A, De Rycke Y, Dalichampt M, et al. Reduced risk of cancer among low-dose aspirin users: data from French health care databases. Pharmacoepidemiol Drug Saf 2019;28:1258-66.

29 Cook NR, Lee I-M, Zhang SM, et al. Alternate-Day, low-dose aspirin and cancer risk: long-term observational follow-up of a randomized trial. Ann Intern Med 2013;159:77-85.

30 Elwood PC, Morgan G, Galante J, et al. Systematic review and meta-analysis of randomised trials to ascertain fatal gastrointestinal bleeding events attributable to preventive low-dose aspirin: no evidence of increased risk. PLoS One 2016;11:e0166166. 